Briefs July 1995). Doses of 0.3 to $0.6 \mathrm{mg} / \mathrm{kg}$ were recommended in clinical practice, larger doses having little advantage and causing possible impairment of cognitive functioning with multiple daily doses.

\title{
TREATMENT-CONTINUITY OF ADHD COMPARED USING IMMEDIATE-RELEASE AND EXTENDED-RELEASE MPH
}

The continuity of methylphenidate (MPH) therapy for ADHD in young Medicaid beneficiaries (ages 6 to 17 years) treated with immediate-release (IR) or extended-release (ER) MPH formulations was compared in an analysis of statewide California Medicaid claims (2000-2003) conducted at Columbia University, New York; University of Pennsylvania, Philadelphia; and McNeil Pharmaceuticals, Fort Washington, PA. Compared to IR-MPH treatment, patients initiating ER-MPH had a significantly longer mean duration of treatment (140.3 days vs 103.4 days). Controlling for group differences in age, sex, and other factors, ER-MPH-treated patients had an average $37 \%$ longer duration of treatment than those receiving IR-MPH. Comparing ER-MPH preparations, Concerta treatment was continued longer than Metadate CD or Ritalin LA (147.2, 113.0, and 101.1 days, respectively). (Marcus SC, Wan GJ, Kemner JE, Olfson M. Continuity of methylphenidate treatment for attention-deficit/hyperactivity disorder. Arch Pediatr Adolesc Med June 2005;159:572-578). (Respond: Mark Olfson MD MPH, New York State Psychiatric Institute/Department of Psychiatry, College of Physicians and Surgeons of Columbia University, 1051 Riverside Dr, New York, NY 10032).

COMMENT. In the above population, ER-MPH formulations for the treatment of $\mathrm{ADHD}$ were associated with longer treatment continuity than IR-MPH. Hispanic and African American Youth and adolescents were more likely to discontinue treatment early than other ethnic groups and younger children. Overall, less than one half of the patients continued MPH therapy beyond 90 days and less than $20 \%$ continued for 1 year. Long-acting MPH preparations appear to prolong compliance and continuity of therapy, and treatment with Concerta (12 hour duration of action) is maintained longer than Ritalin LA that is effective for only 8 hours.

In addition to duration of action, factors important in continuity of therapy include efficacy and toxicity of medication, educational accommodations, counseling, frequency of return visits and availability of professional consuitation, and cultural bias. When therapy is interrupted at weekends and during vacation periods, and medication is used as an aid to education during school term, the overall duration of therapy is frequently extended through childhood and into adolescence, depending on the response and need.

In an overview and analysis of the current literature on the treatment of ADHD, the AAP Committee on Quality Improvement, Subcommittee on ADHD concluded that: 1) ADHD should be managed as a chronic condition; 2) the use of stimulant medications is beneficial and that stimulants are equally effective; 3 ) behavioral therapy is minimally effective but only in combination with medication; and 4) education and counseling of patient and family are necessary adjuncts to drug therapy. (Brown RT et al. Pediatrics June 2005;115:e749-e757). 